\title{
Analysis on Load Torque Effect for Assistive Robotic Arms
}

\section{Byoung-Ho Kim}

Department of Mechatronics Engineering, Kyungsung University, Busan, Korea

\section{]jfis}

\begin{abstract}
This paper analyses the load torque effect for assistive robotic arms. For the purpose, we consider a typical assistive robotic arm delivering some loads and derive the torque effect at the joint space of the robot arm. We also try to find the reliable torque range of each joint for exemplary load manipulation tasks. Thus the available torque patterns and ranges of the shoulder and elbow joints have been identified by various simulations and discussed the load effects for the delivering tasks by assistive robotic arms. As a result, this analysis is practically helpful for us to determine the specifications of actuators to be employed at the joint space of humanoid, prosthetic and industrial robotic arms. It is also applicable for the purpose of effective rehabilitation of human arms using some loads.
\end{abstract}

Keywords: Torque analysis, Shoulder and elbow joints, Assistive robotic arm, Rehabilitation

\section{Introduction}

When dealing with some loads in the human space, it is desirable to use an assistive robotic arm. Of course, it may be more advantageous to use a human arm in the case of simple tasks. Even though a simple delivering task, it will be a hard work if a worker have to do such a work repeatedly for a long time, and its work performance will be decreased. In particular, it is well known as a very hard work to dealing with a heavy patient in a hospital. Thus we need to develop a proper assistive robotic arm that can support those works. It is also an interesting issue to research such a robotic arm for people whose arms are uncomfortable [1, 2]. Especially it is important to consider a combined motion of lifting and transferring for effective handling of an object.

Recently, many researchers are trying to develop a humanoid robot with dexterous arms that is applicable for various object manipulation tasks [3, 4]. Many valuable researches to design an effective arm mechanism have been performed and their results will be of great contribution to various object handling tasks [5, 6]. It is also remarkable that many dangerous field works can be performed based on a coordination of human-like robotic arms and mobility [7, 8]. On the other hand, some researchers have a concern to develop an effective exoskeleton arm mechanism for the purpose of rehabilitation or replacement of human arms [9, 10]. Some researches on an artificial prosthetic arm are also being conducted actively for disabled persons who have arm motions limited by accidents [11]. In this paper, we focus on the fundamental torque characteristics of the shoulder and elbow joints of such a robotic arm that should be carefully considered for effective load manipulation [12]. 
Practically, proper selection of adequate actuators is a fundamental step in the design procedure of such a robotic arm and plays an important role for setting the available payload. It is because the torque requirements of all joints should be guaranteed to ensure the payload of the arm mechanism for given tasks. Therefore it is required to confirm that the torque ranges of all joint actuators used in the robot arm are available for the payload expected in the work space. It is also noticeable that the torque patterns of those joints are very important factors to get a good performance according to the applications of the robot arm [13]. This observation is significant in terms of biomechanical movement for effective rehabilitation of the shoulder and/or elbow joints of human arms [12, 14]. Specifically, analysis on the torque characteristics of the shoulder and elbow joints of a humanoid robot arm is an interesting issue for its application to various industrial and human works. Thus, we had a concern to present those torque characteristics in handling an object [15].

The objective of this paper is to provide a more comprehensive analysis for effective load manipulation by using an assistive robotic arm based on the previous work. We additionally address on the torque balance in the sequential multiple load manipulation work and its motion effect for the purpose of rehabilitation works. This paper is organized as follows: in Section 2, we specify a load handling task using an assistive robotic arm and try to derive the joint torque relations of the robotic arm for the payload. In Section 3, we perform various simulations to show the torque patterns and reliable ranges of the shoulder and elbow joints. Finally, concluding remarks are drawn in Section 4.

\section{Model of Load Handling Tasks}

A load handling task by using an assistive robotic arm is modeled in this section for the purpose of analysing the load effects at the shoulder and elbow joints, and we describe the torque relations of those joints to perform typical lifting and transferring tasks.

Figure 11.a) shows a common handling task of some books by a human arm. If the load is heavy, we need to use an assistive robotic arm of a mobile manipulation system. It is well known that the human arm can be modeled as a robotic mechanism with seven-degrees of freedom which works in the three-dimensional space in a natural fashion [1]. In this paper, however, we simplify it as an assistive robotic arm with three-degrees of freedom, as shown in Figure 1 b), which is available to manipulate an object in the two-dimensional space. It has a shoulder joint, an

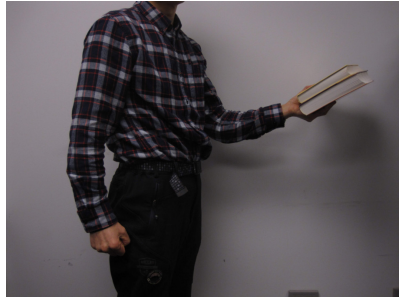

(a)

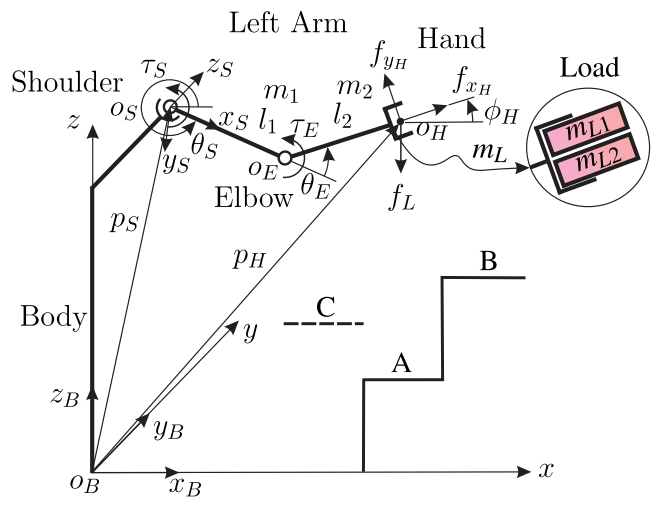

(b)

Figure 1. Load handling tasks by using (a) a human arm and (b) an assistive robotic arm.

elbow joint, and a simple gripper mechanism. So it can be used to grasp some loads and transfer them from the place A to B, or $\mathrm{C}$ in the $x z$-plane of Figure 1 (b) by combining the motions of the shoulder and elbow joints.

The dynamic equation of the assistive robotic arm in Figure 1 (b) is generally represented by the following form at the joint space [16]:

$$
\boldsymbol{I}_{\phi \phi}^{*} \ddot{\phi}+\dot{\phi}^{T} \boldsymbol{P}_{\phi \phi \phi}^{*} \dot{\phi}+\tau_{\phi L}=\tau_{\phi},
$$

where $\tau_{\phi} \in R^{n_{\phi} \times 1}\left(n_{\phi}:\right.$ the degree of freedom for the arm joints) denotes the control torque vector at the joint space. The additional joint torque vector $\tau_{\phi L}$ implies the joint torque effect influenced by the external forces and moments. The notations of $\boldsymbol{I}_{\phi \phi}^{*}$ and $\boldsymbol{P}_{\phi \phi \phi}^{*}$ imply the effective inertia matrix and the inertia power array, respectively, that induce the self-load effects by the mass, $m_{1}$ and $m_{2}$, of each link of the robot arm.

For an object manipulation by using such an assistive robotic arm, it is necessary to consider not only the weight of the arm itself, but also the additional torque effects required to support the external loads. However, we focus on the pure joint torque effects affected by the load of objects grasped in the hand space. This viewpoint is very useful for evaluating the load effects in delivering tasks by assistive robotic arms as well as in various 
exercises of rehabilitation by using some loads. So the self-load terms in (1) have been excluded in this analysis, and thus the torque characteristics of all joints to support the loads handled can be effectively shown.

In fact, the control torque vector at the joint space of the robot arm shown in Figure 1 b) can be represented by

$$
\tau_{\phi}=\left[\begin{array}{cc}
\tau_{S} & \tau_{E}
\end{array}\right]^{T},
$$

where $\tau_{S}$ and $\tau_{E}$ denote the shoulder and elbow joint torques, respectively. And the additional joint torque vector can be expressed by

$$
\begin{aligned}
\tau_{\phi L} & =\left[\begin{array}{ll}
\tau_{S L} & \tau_{E L}
\end{array}\right]^{T} \\
& =\left[\boldsymbol{G}_{\phi}^{h}\right]^{T} f_{H}
\end{aligned}
$$

that reflects the external load force, $f_{H}$, required for handling the loads. The two components of $\tau_{S L}$ and $\tau_{E L}$ represent the shoulder and elbow joint torques, respectively, considering the influence of the external load. The kinematic Jacobian matrix relating the hand space to the joint space of the arm $\left[\boldsymbol{G}_{\phi}^{h}\right]^{T}$ is determined by

$$
\left[\boldsymbol{G}_{\phi}^{h}\right]^{T}=\left[\begin{array}{cc}
l_{1} \sin \theta_{E}(t) & l_{1} \cos \theta_{E}(t)+l_{2} \\
0 & l_{2}
\end{array}\right],
$$

where $l_{i}(i=1,2)$ denotes the length parameters of the robot arm. The parameters of $\theta_{S}(t)$ and $\theta_{E}(t)$ represent the shoulder and elbow joint angles and those angles can be planned by the operational trajectory desired at the hand space $o_{H}$.

The external load force vector $f_{H}$ is given by

$$
f_{H}=\left[f_{x_{H}}(t) f_{y_{H}}(t)\right]^{T},
$$

where the $x$ - and $y$-directional force components at the hand space, $f_{x_{H}}(t)$ and $f_{y_{H}}(t)$, can be determined by

$$
\begin{aligned}
& f_{x_{H}}(t)=\sin \left(\theta_{S}(t)+\theta_{E}(t)\right) f_{L}, \\
& f_{y_{H}}(t)=\cos \left(\theta_{S}(t)+\theta_{E}(t)\right) f_{L}, \\
& f_{L}=-m_{L} g
\end{aligned}
$$

where $f_{L}$ denotes the load force activated by the multiple objects. The parameter of $m_{L}$ denotes the mass of load, and $g$ is the acceleration of gravity. In general, many loads can be additionally considered in this form.

According to literatures [13, 17], the typical movement pat- terns of a load by human arms can be empirically classified as follows: 1) vertical lifting to the $z$-direction in Figure 1(b), 2) horizontal transferring to the $x$-direction on a constant vertical level, 3) horizontal transferring to the $y$-direction on a constant vertical level, 4) vertical lifting and horizontal transferring in a two-dimensional space, e.g. the $x z$ plane, and 5) general movement in the three-dimensional space. Especially, the fourth case can be considered as a basic pattern of an object manipulation and this pattern is commonly used for warming up of the shoulder and elbow joints of human arms in many sports activities. To be specific, in order to deliver an object from the position A to B in Figure 1 (b), a proper combination of a lift action to the $z$-axis direction and a transfer motion to the $x$-axis direction is practically required. Thus we try to consider such a pattern to identify the torque patterns and ranges of the shoulder and elbow joints in handling some loads by using the assistive robot arm.

\section{Simulation and Analysis}

This section reveals various simulations and analyses on the torque patterns and ranges of the shoulder and elbow joints to support the object load handled by an assistive robotic arm.

For our simulation studies, we employed the robot arm shown in Figure 1 (b) that is maneuverable with some loads in the $x z$ plane. The length parameters of the upper and lower links of the robot arm have been assigned as $0.30 \mathrm{~m}$ and $0.33 \mathrm{~m}$, respectively by measuring the corresponding lengths of the author. For effective identifying those torque characteristics, we performed various simulations based on the following two scenarios: 1) constant load handling, where the robot arm delivers a constant load through the overall trajectory defined in the operational space, and 2) varying load handling, where the robot arm moves for a while under a load assigned initially, and then it has to adapt a load change during moving to the end position. The objective of the first scenario is to show the torque patterns and ranges of the shoulder and elbow joints when the robot arm transfers a constant load through the given trajectory. The second scenario is to confirm the weighting effects of those joints as the load is varied. The point mass of each load regardless of the shape is only considered in our simulations.

For the first scenario, a constant load of $5 \mathrm{kgf}$ has been assigned. The operational trajectory of the load has been planned based on the origin of the shoulder, and it has been classified as two phases, lift and transfer, like the fourth pattern presented in the previous section. The operational trajectory of the load for 
Table 1. Parameters for object lifting and transferring tasks

\begin{tabular}{|c|c|c|c|c|c|}
\hline Case & $A_{x}(\mathrm{~m})$ & $A_{z}(\mathrm{~m})$ & $d_{x}(\mathrm{~m})$ & $d_{z}(\mathrm{~m})$ & Tasks \\
\hline 1 & 0.30 & & & & lift up and \\
\hline 2 & 0.35 & 0.20 & 0.15 & 0.20 & outward \\
\hline 3 & 0.40 & & & & (extension) \\
\hline 4 & 0.30 & & & & lift up and \\
\hline 5 & 0.35 & 0.20 & -0.15 & 0.20 & inward \\
\hline 6 & 0.40 & & & & (flexion) \\
\hline
\end{tabular}

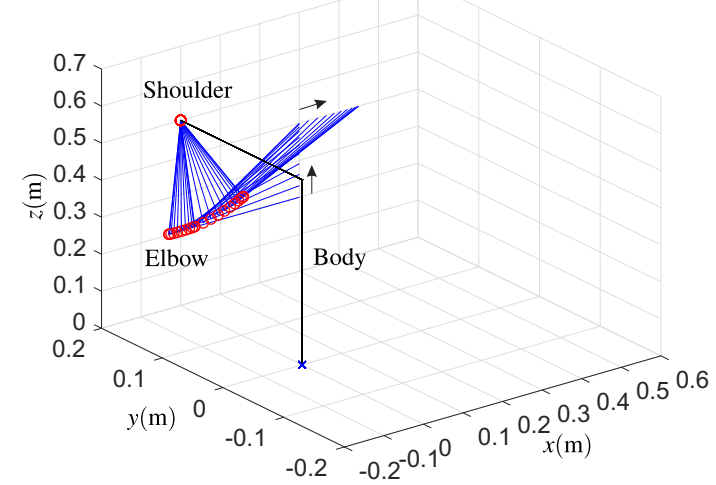

Figure 2. Trajectory of the assistive robotic arm for the first simulation (Case 1).

the lift phase is determined by

$$
\left.\begin{array}{l}
x_{H}(t)=A_{x}, \\
z_{H}(t)=A_{z}+d_{z} \sin 0.5 \pi t,
\end{array}\right\} 0 \leq t \leq 1.0 .
$$

The operational trajectory of the load for the transfer phase is given by

$$
\left.\begin{array}{l}
x_{H}(t)=A_{x}+d_{x} \sin 0.5 \pi(t-1.0), \\
z_{H}(t)=A_{z}+d_{z}
\end{array}\right\} 1.0 \leq t \leq 2.0 .
$$

In fact, based on the trajectory planning, the object is to be lifted from the initial $z$-axis position $\left(A_{z}\right)$ to the $z$-axis direction for one second at a fixed position of $A_{x}$ on the $x$-axis. After that, during the remaining one second, the object is to be transferred from the initial $x$-axis position $\left(A_{x}\right)$ to the target position on the $x$-axis direction while maintaining the current $z$-axis position. Thus, various trajectories for such a delivering task can be assigned by selecting those parameters of $A_{x}, d_{x}, B_{z}$ and $d_{z}$. For effective analysis, we assigned those parameters as Table 1

The first task of this simulation (Case 1) is to lift and transfer the object from the coordinate of $x y z(0.3,0.2,0.2)$ to $x y z(0.45$, $0.2,0.4)$ as shown in Figure 2 which represents the overall vestige diagram of the assistive robot arm.

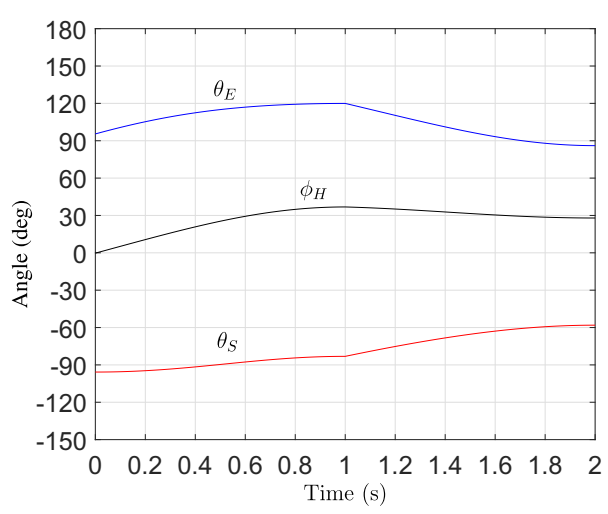

(a)

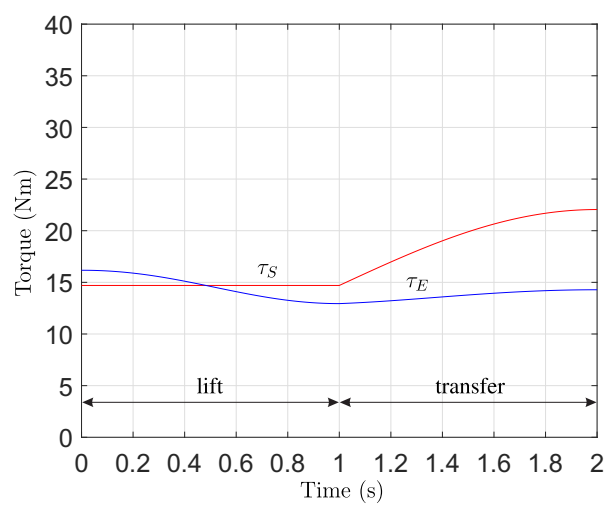

(b)

Figure 3. Trajectories of the shoulder and elbow joints in the first simulation (Case 1) and their torque profiles to support the load during the lift and transfer phases. (a) Joint trajectories and (b) joint torques.

Figure 3 (a) shows the resultant trajectories of the shoulder joint $\left(\theta_{S}\right)$ and the elbow joint $\left(\theta_{E}\right)$ to follow the operational trajectory of the load, It is found that the shoulder and elbow joint angles are gradually increased in the lift phase. In the transfer phase, the shoulder joint angle increases, but the elbow joint angle decreases. This joint profiles can be easily identified empirically from the motion of the human arm. The trajectory of $\phi_{H}$ represents the orientation of the load grasped at the hand space that means the current posture of the load. Figure 3 (b) represents the torque characteristics of the shoulder joint $\left(\tau_{S}\right)$ and the elbow joint $\left(\tau_{E}\right)$ required to support the load during the lift and transfer phases. In particular, it is interestingly remarkable that the elbow joint torque for this task is changing for the overall trajectory to sustain the load, but the shoulder torque is not changing especially during the lift phase. That is, it stays at a certain level which is actually determined at the initial state. This is actually an interesting tendency in such a load delivering task. If the self-load terms in (1) are simultaneously considered in this simulation, this weighting 


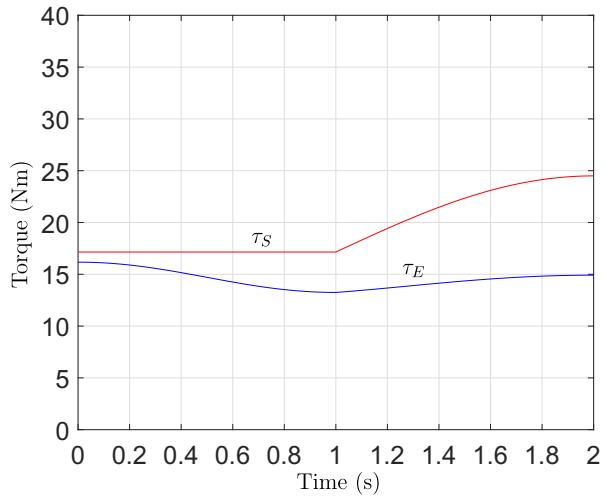

(a)

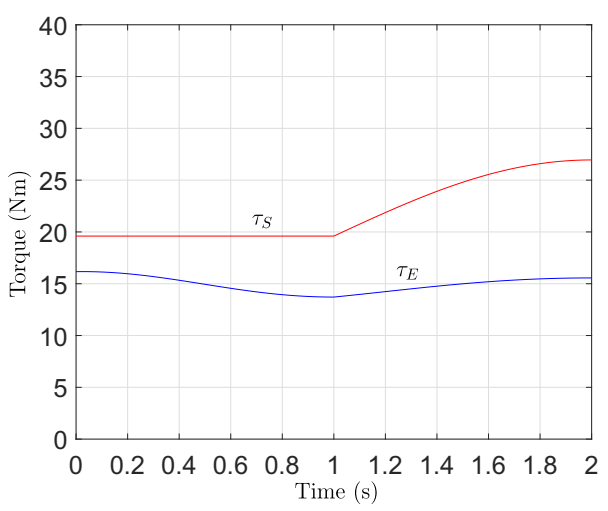

(b)

Figure 4. Torque profiles of the shoulder and elbow joints in the second and third simulations. (a) Case 2 and (b) Case 3.

effect at the shoulder joint may not be revealed clearly.

In order to verify the shoulder torque's trend found in Figure 3 b), we performed similar simulations given by the second and third cases in Table 1 It is really an interesting point to observe the shoulder torque value according to the starting position of the load handled by the robot arm.

From Figure $4(a)$ and $4(b)$, we can find that the torque value of the shoulder joint during the lift phase is different according to the starting position, but it stays in each constant level keeping with the initial torque value. And the elbow joint torque during the lift phase is mitigated gradually in the given workspace. As a result, we can recognize that the shoulder joint torque to support the load assigned in such a lifting task depends on the starting position of the load, but there is no need to consider additional torque in the process of such a vertical motion. On the other hand, it is confirmed that both torque values in the transfer phase for the horizontal motion tend to be increased as the starting position is far from the origin of shoulder. This means that more harder load can be applied to those joints according to the distance. Specifically, it is shown

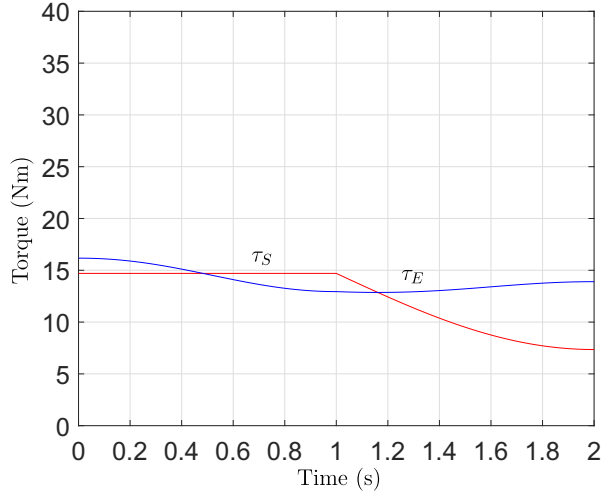

(a)

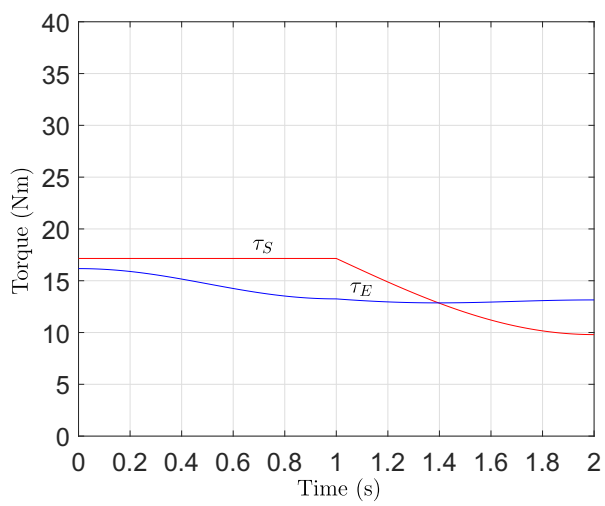

(b)

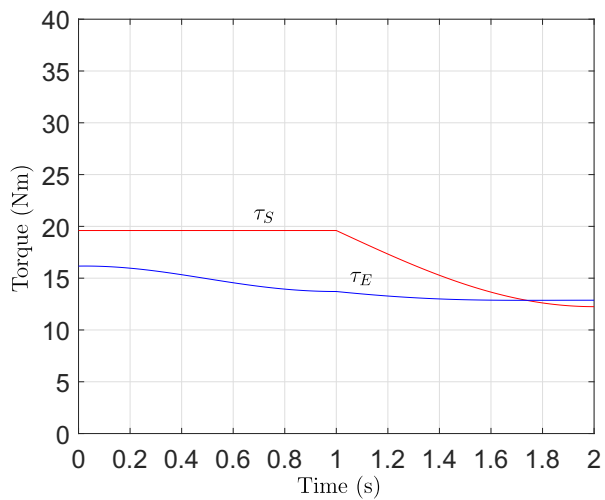

(c)

Figure 5. Torque profiles of the shoulder and elbow joints for the motion of flextion. (a) Case 4, (b) Case 5, and (c) Case 6.

that the shoulder joint torque is more bigger than the elbow joint torque in the extension process. This estimation is helpful for selecting a proper size of any actuator used in the design of such an assistive robotic arm.

The next three simulations (Cases 1-3) have been intended to identify the torque characteristics of the shoulder and elbow joints to support the load through the motion trajectory of flexion of such an assistive robotic arm. 
Figure 5(a) shows the torque characteristics of the shoulder and elbow joints required to support the load through the fourth trajectory. That is, the shoulder torque tends to be gradually decreased in the horizontal inward motion compared to the outward motion of the first simulation as shown in Figure 3 (b). Also, the torque characteristics of the shoulder and elbow joints in the fifth and sixth simulations have been shown in Figure 5 (b) and 5(c), respectively. They are very similar to the pattern shown in Figure 5 (a), but the overall shoulder torques become more larger as the starting position of the load is far from the origin of shoulder. It is empirically acceptable likewise the cases of the extensional object manipulation.

Next, for the second scenario handling a varying load, we reconsidered the third and sixth simulations by changing the load condition according to time. The assigned arm motion and the load condition are as follows. The robot arm moves for 0.6 seconds under a load of $5 \mathrm{kgf}$, and then moves for 0.8 seconds by adding $2 \mathrm{kgf}$. After 1.4 seconds, the robot puts the added load down and delivers the initial load to the target position. This is actually to confirm the weighting effect of the shoulder and elbow joints for assistive robotic delivering tasks.

Figure 6 a) and 6 b) show the torque characteristics of the shoulder and elbow joints to support such a varying load through the operational trajectories defined in the Case 3 and Case 6, respectively. In fact, they are basically similar to the patterns shown in Figure 4(b) and Figure 5(c), respectively, but those torque values become larger when the additional load is involved. Thus we can check that the shoulder and elbow joint torques to support safely the varying load should be at least greater than $34 \mathrm{~N} \cdot \mathrm{m}$ and $21 \mathrm{~N} \cdot \mathrm{m}$, respectively. This effort is practically valuable for an engineer to determine the specification of any actuator in the design process of assistive robotic arms.

Thus, in order to provide a guideline in determining a certain sufficient joint torque range for such a load handling task, we reconsidered the third simulation by considering various loads based on the first scenario. It is because more larger joint torque is required in the third extensional delivering process as shown Figure 3 b). The minimum requirements in the torque values of the shoulder and elbow joints to perform this task safely have been specified in Table 2. It is remarkable that for the given extensional task to deliver up to $10 \mathrm{kgf}$, we need to consider at least $53.90 \mathrm{~N} \cdot \mathrm{m}$ and $32.34 \mathrm{~N} \cdot \mathrm{m}$ as an additional torque capability of the shoulder and elbow joint actuators besides their torques for the self-load of the robot arm. This guideline is fundamentally important for a safe object manipulation.

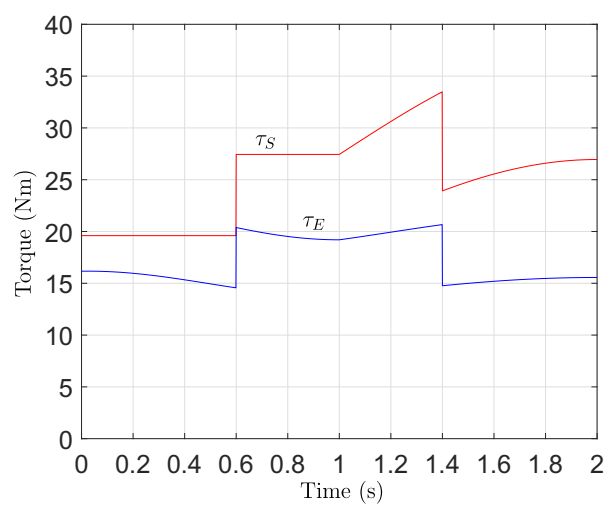

(a)

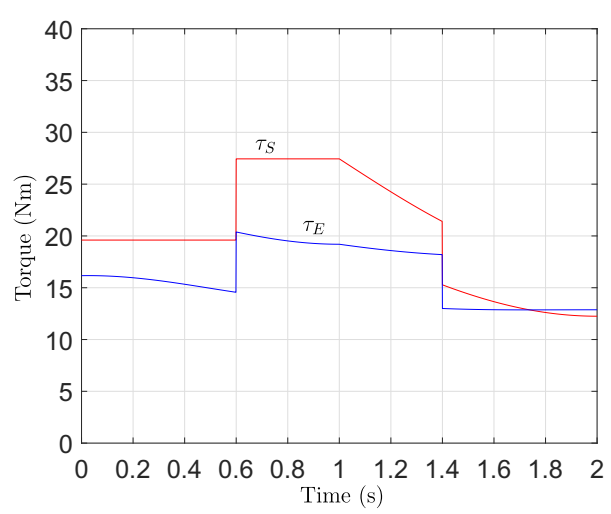

(b)

Figure 6. Torque profiles of the shoulder and elbow joints to support varying load. (a) Case 3 and (b) Case 6.

Table 2. Minimum requirements in the torque values of the shoulder and elbow joints for the given task

\begin{tabular}{cccc}
\hline$m_{L}(\mathrm{kgf})$ & $\tau_{S}(\mathrm{~N} \cdot \mathrm{m})$ & $\tau_{E}(\mathrm{~N} \cdot \mathrm{m})$ & Tasks \\
\hline 0 & 0 & 0 & Case 3 \\
1 & 5.39 & 3.23 & $:$ extensional delivering \\
2 & 10.78 & 6.47 & based on \\
3 & 16.17 & 9.70 & the first scenario \\
4 & 21.56 & 12.94 & \\
5 & 26.95 & 16.17 & \\
6 & 32.34 & 19.40 & \\
7 & 37.73 & 22.64 & \\
8 & 43.12 & 25.87 & \\
9 & 48.51 & 29.11 & \\
10 & 53.90 & 32.34 & \\
\hline
\end{tabular}

In addition, we can see that the greater joint torques are required as the weight of the object is larger, but their patterns may different according to the given delivering task. This trend can make a positive motion effect for the purpose of 
rehabilitation of human arms. That is, by using this analysis, various operational trajectories can be tested while confirming those joint torques according to the purpose of rehabilitation. In this point of view, this study can give a preliminary guideline for a rehabilitation doctor to make a proper motion effect for effective arm rehabilitations [12].

Through the simulations, we specified the torque patterns and ranges of the shoulder and elbow joints for typical object delivering tasks by using an assistive robotic arm. The shoulder joint torque to support the object load is basically dependent on the distance between the origin of the shoulder and the hand space. For any vertical motion, however, it tends to be maintained at a constant level determined at the initial posture. This means that further shoulder torque is practically not necessary in the process of such a vertical life motion. On the other hand, it is noticeable in terms of exercise that such a vertical lift motion does not generate additional training effect on the shoulder joint except the initial load effect. The overall torque of the elbow joint is also dependent on the distance, but such an effect is not generated in the elbow motion.

As a result, in terms of design aspect, this analysis can be applied to identify a proper torque pattern and range of each joint of an assistive robotic arm handling an object. The specified joint torque ranges can be used for effective selection of appropriate joint actuators for assistive robotic arms handling an object. Also, this analysis can be applied to establish a personalized rehabilitation plan for human arms and develop a rehabilitation or medical robotic arm [2, 18]. Of course, this analysis can be expanded to three-dimensional works, where the self-load of a robotic arm could be considered.

\section{Concluding Remarks}

This paper presented the torque characteristics of the shoulder and elbow joints for load manipulation tasks using an assistive robotic arm which has been modeled based on a load handling task by human arms. Through the exemplary load delivering tasks, we showed the available torque profiles and ranges of those joints to support the external load at the operational space and analysed their effects on arm motions. Also, the operating trajectories and the weighting effect of multiple loads have been discussed.

As a result, the available joint torque ranges of assistive robotic arms to support the operational load can be verified based on this analysis in advance. Thus it can be easy to determine the specification of any actuator for an assistive robotic arm in the design aspect. It is also confirmed that the torque patterns and ranges of the shoulder and elbow joints can be greatly influenced by the operating trajectory of the load and its weight. The elbow joint torque for the given tasks has been identified as a kind of normal pattern through the overall trajectories of the load. Its size depends on the distance between the shoulder space and the operational space. However, the shoulder torque pattern showed a different tendency according to the lift phase or the transfer phase. That is, the shoulder joint torque in the vertical lifting motion keeps the torque value determined by the weight of the load at the initial arm posture. On the other hand, the shoulder torque during the horizontal transfer process becomes larger for extension or smaller for flexion, and its level is also dependent on the distance from the shoulder space to the operational space. This observation can give us a guideline to plan a safe operational range for object manipulation tasks by using assistive robotic arms. Also, in the viewpoint of arm rehabilitation, this study is applicable for a rehabilitation doctor to determine an adequate load for effective training of the shoulder joint and/or the elbow joint.

Additional analysis for various tasks coordinated by two arms will be an interesting issue.

\section{Conflict of Interest}

No potential conflict of interest relevant to this article was reported.

\section{Acknowledgement}

This research was supported by Kyungsung University Research Grants in 2018.

\section{References}

[1] J. C. Perry and J. Rosen, "Design of a 7 degree-of-freedom upper-limb powered exoskeleton," in Proceedings of the 1st IEEE/RAS-EMBS International Conference on Biomedical Robotics and Biomechatronics, Pisa, Italy, 2006, pp. 805810. http://doi.org/10.1109/BIOROB.2006.1639189

[2] Z. Song and S. Guo, "Design process of exoskeleton rehabilitation device and implementation of bilateral upper limb motor movement," Journal of Medical and Biological Engineering, vol. 32, no 5, pp. 323-330, 2012.

[3] Y. Sakagami, R. Watanabe, C. Aoyama, S. Matsunaga, N. Higaki, and K. Fujimura, "The intelligent ASIMO: system 
overview and integration," in Proceedings of IEEE/RSJ International Conference on Intelligent Robots and Systems, Lausanne, Switzerland, 2002, pp. 2478-2483. http://doi.org/ 10.1109/IRDS.2002.1041641

[4] I. Mizuuchi, T. Yoshikai, Y. Sodeyama, Y. Nakanishi, A. Miyadera, T. Yamamoto, et al., "Development of musculoskeletal humanoid Kotaro," in Proceedings of IEEE International Conference on Robotics and Automation, Orlando, FL, 2006, pp. 82-87. http://doi.org/10.1109/ROBOT.2006. 1641165

[5] J. S. Mehling, P. Strawser, L. Bridgwater, W. K. Verdeyen, and R. Roverkamp, “Centaur: NASAs mobile humanoid designed for field work,' in Proceedings of IEEE International Conference on Robotics and Automation, Rome, Italy, 2007, pp. 2928-2933. http://doi.org/10.1109/ROBOT. 2007.363916

[6] C. Ott, O. Eiberger, W. Friedl, B. Bauml, U. Hillenbrand, C. Borst, et al., "A humanoid two-arm system for dexterous manipulation," in Proceedings of 6th IEEE-RAS International Conference on Humanoid Robots, Genova, Italy. 2006, pp. 276-283. http://doi.org/10.1109/ICHR.2006. 321397

[7] National Aeronautics and Space Administration, "Robonaut 1: a humanoid robot, Centaur," 2006. Available https://robonaut.jsc.nasa.gov/R1/field-ops/desert06.asp

[8] Neobotix, "Mobile manipulator MM-500," 2018. Available https://www.neobotix-robots.com/mobile-manipulatormm-500.html

[9] J. C. Perry, J. Rosen, and S. Burns, "Upper-limb powered exoskeleton design," IEEE/ASME Transactions on Mechatronics, vol. 12, no. 4, pp. 408-417, 2007. http: //doi.org/10.1109/TMECH.2007.901934

[10] Y. Bae, H. Y, Choi, and Y. Moon, "The design of robot arm based on the morphological, neurological model of human," Journal of Korean Institute of Intelligent Systems, vol. 17, no. 4, pp. 500-505, 2007. https://doi.org/10.5391/ JKIIS.2007.17.4.500

[11] S. K. Kundu and K. Kiguchi, "Development of a 5 DOF prosthetic arm for above elbow amputees," in Proceedings of 2008 IEEE International Conference on Mechatronics and Automation, Takamatsu, Japan, 2008, pp. 207-212. https://doi.org/10.1109/ICMA.2008.4798753
[12] M. H, Rahman, T. K, Ouimet M. Saad, J. P. Kenne and P. S. Archambault, "Development and control of a wearable robot for rehabilitation of elbow and shoulder joint movements," in Proceedings of the 36th Annual Conference of the IEEE Industrial Electronics Society, Glendale, AZ, 2010, pp. 1506-1511. https://doi.org/10.1109/IECON.2010. 5675459

[13] J. Watkins, Structure and Function of the Musculoskeletal System. Champaign, IL: Human Kinetics, 1999.

[14] A. Freivalds, Biomechanics of the Upper Limbs: Mechanics, Modeling, and Musculoskeletal Injuries. Boca Raton, FL: CRC Press, 2004

[15] B. H, Kim, "Torque characteristics of shoulder and elbow joints of assistive robotic arms handling an object," in Proceedings of 2016 6th IEEE International Conference on Biomedical Robotics and Biomechatronics (BioRob), Singapore, 2016, pp. 26-29. https://doi.org/10.1109/BIOROB. 2016.7523819

[16] R. A. Freeman and D. Tesar, "Dynamic modeling of serial and parallel mechanisms/robotics systems. Part I. Methodology," in Trends and Development in Mechanisms, Machines, and Robotics 1988. New York, NY: American Society of Mechanical Engineers, 1988.

[17] V. M. Zatsiorsky, Kinetics of Human Motion. Champaign, IL: Human Kinetics, 1998.

[18] Control System Technology Co. Ltd., "Elbow CPM," http //www.cmcst.co.kr

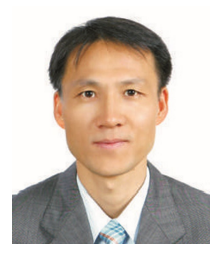

Byoung-Ho Kim received the Ph.D. in the Department of Electronic Engineering from Hanyang University in 2001. Dr. Kim is currently with the Department of Mechatronics Engineering, Kyungsung University, Busan, Korea as a professor since 2005. His research interests include assistive robotic arms, biomimetic system modeling, multi-fingered hands, soft manipulation, mobile manipulation, intelligent control for humanoids, health care mechanisms, rehabilitation and medical science.

E-mail: kimbh@ks.ac.kr 